\title{
Desarrollo físico y nutrición de preescolares habaneros según nuevos patrones de crecimiento de la OMS
}

\section{Physical development and nutritional status of preschool-age children in Havana according to the new WHO Growth References}

\author{
Mercedes Esquivel Lauzurique'; Ciro González Fernández"
}

'Doctora en Ciencias Médicas. Facultad de Ciencias Médicas "Julio Trigo López."
Instituto Superior de Ciencias Médicas de La Habana. La Habana, Cuba.
"Máster en Estadística Aplicada. Facultad de Ciencias Médicas "Julio Trigo López."
Instituto Superior de Ciencias Médicas de La Habana. La Habana, Cuba.

\section{RESUMEN}

Objetivos Evaluar el desarrollo físico y estado nutricional de preescolares habaneros mediante su comparación con los nuevos patrones de crecimiento de la OMS.

Métodos Se estimaron las puntuaciones $Z$ de los indicadores peso/edad, talla/edad, peso/talla e índice de masa corporal/edad en 1322 preescolares que integraron la muestra del Estudio sobre Crecimiento y Desarrollo de Ciudad de La Habana, 2005. Se calcularon las medias y desviaciones estándar de estas puntuaciones y se estimaron las tasas de prevalencia de insuficiencia ponderal, cortedad de talla, emaciación y sobrepeso. Se compararon estos últimos resultados con los que se obtendrían con el uso de la antigua referencia de la OMS y con las normas nacionales cubanas.

Resultados Los valores medios de las puntuaciones $Z$ resultaron positivos, en el rango entre 0,33 y 0,51 , sólo la talla para la edad mostró un valor negativo de 0,07 . En los indicadores relacionados con el peso, los valores de esas puntuaciones mostraron una tendencia ascendente hasta los dos años, posteriormente, adquirieron un comportamiento descendente, mientras que la talla mostró cifras 
iniciales negativas seguidas, después de los dos años, por valores cercanos a cero o francamente positivos. Las prevalencias de insuficiencia ponderal, cortedad de talla y emaciación, fueron de 1,$3 ; 3,7 ; 1,0 ;$ y $1,0 \%$, respectivamente. El porcentaje de niños con sobrepeso fue de $11,2 \%$ con el índice de masa corporal/edad y de 10,7 $\%$ con el peso/talla. Se detectaron amplias variaciones en la prevalencia de las diferentes formas de malnutrición con el uso de normas de referencia.

Conclusiones Los resultados de la evaluación del crecimiento y estado de nutrición de los niños dependen, fundamentalmente, de la referencia utilizada; las diferencias encontradas son relevantes y también lo son las implicaciones que tienen sobre las políticas y estrategias a desarrollar en la atención a esta población.

Palabras clave: Antropometría, referencias de crecimiento, monitoreo del crecimiento, peso, talla.

\section{ABSTRACT}

Objectives To assess the physical development and nutritional status of preschoolage children living in Havana through the comparison with the new World Health Organization's Growth Reference.

Methods The $z$ scores of weight/age, height/age, weight/height, and body mass index/age of 1322 children aged 0-4 years were estimated in the Havana's 2005 Growth and Development Study. Means and standard deviations of the z scores and the rates of prevalence of underweight, stunting, wasting and overweight were calculated.. These last results were compared with those that would have been obtained with the use of the prior WHO Growth References and with the Cuban domestic standards.

Results Mean values of $z$ scores were positive in the range from 0.33 to 0.51 , except for the height/age that reached a negative value of $-0,07$. In the case of weight indicators, the $z$ scores increased their values up to two years of age and then they declined, whereas height/age began with negative values and, after the age of 2 years, it showed close to zero or positive values. The prevalence rates of underweight, stunting and wasting were $1.3 \%, 3.7 \%, 1.0 \%$ and $1.0 \%$ respectively. The percentage of overweight among children was $11.2 \%$ with body mass index/age and $10.7 \%$ with weight/height. Significant variations in the prevalence of different forms of malnutrition were detected with the use of growth reference standards.

Conclusions The results of the assessment of growth and nutritional status of children depend fundamentally on the type of references used; the differences found are relevant and also their implications on the policies and strategies to be developed for the care of this population.

Key words: Anthropometry, growth references, growth monitoring, weight, height.

\section{NTRODUCCI ÓN}

El interés por el desarrollo integral de los niños ha crecido en todo el mundo como resultado del incremento constante de la supervivencia infantil y el reconocimiento 
de que las experiencias de la infancia ejercen efectos considerables en la futura capacidad funcional del ser humano. ${ }^{1} \mathrm{~A}$ ello se añade el hecho de que numerosos organismos internacionales descansan en la evaluación del crecimiento de los niños para medir el bienestar general de las poblaciones y formular políticas de salud ya que es necesario que muchos procesos fisiológicos ocurran normalmente y muchas necesidades sean satisfechas desde la vida fetal y en la infancia para que este ocurra de manera adecuada. ${ }^{2}$

Cuba no escapa de ese interés, y por ello, desde los inicios de la década de los 70 y a la par del inicio del Programa de Reducción de la Mortalidad Infantil en el país, inició la ejecución de estudios antropométricos masivos y periódicos de los niños y adolescentes como una práctica permanente del sistema de salud cubano para conocer los cambios y tendencias ocurridas en el crecimiento de la población de esas edades.

Para el monitoreo del desarrollo físico de los niños resulta necesario el uso de valores de referencia locales o internacionales que ayuden a determinar si el crecimiento de un individuo o grupo poblacional está acorde con lo esperado. En relación con ello, la OMS realizó una encuesta que fue publicada en el 2004 en la que se señalaba que de los 178 países en que se obtuvo información, sólo 25 -entre estos Cuba- utilizaban normas nacionales, el resto usaba las referencias del Centro Nacional de Estadísticas de Salud de los Estados Unidos (NCHS, Nacional Center for Health Statistics) propuestas por la Organización Mundial de Salud (OMS) como referencia para uso internacional, conocidas con las siglas NCHS/OMS. ${ }^{3}$

Los significativos fallos de que adolecían estas referencias motivaron que esa organización estableciera un Grupo de Trabajo sobre Crecimiento Infantil para la obtención de una nueva referencia internacional y el desarrollo de recomendaciones para el uso e interpretación de la antropometría en niños pequeños; debido a ello, en el año 2006 se publicaron los primeros resultados del Estudio Multicéntrico de Referencia del Crecimiento de la OMS, (Multicentre Growth Referente Study, MGRS) brindando así valores de referencia de un conjunto de indicadores antropométricos para la evaluación del desarrollo físico y el estado nutricional de los niños de 0 a 5 años. ${ }^{4,5}$

Se considera por sus autores que esta nueva referencia tiene un carácter prescriptivo, ya que no describe simplemente "cómo crecen los niños en un lugar y momento específico" sino "cómo es el crecimiento de los niños." ${ }^{6}$ El objetivo de este estudio es conocer cómo se comporta el crecimiento de un grupo de menores de 5 años, residentes en la Ciudad de La Habana y que formaron parte de la muestra estudiada en el Estudio Provincial sobre Crecimiento y Desarrollo que se efectuó en esa provincia en el año 2005, al compararlos con los nuevos patrones de la OMS.

\section{MÉTODOS}

El Estudio Provincial sobre Crecimiento y Desarrollo de Ciudad de La Habana, 2005 contó con una muestra probabilística, estratificada y polietápica, de 3935 niños y adolescentes entre 0 y 19 años, residentes en todos los municipios de la ciudad; de ellos 1322 eran menores de cinco años. Para cada uno de los participantes en el estudio se obtuvieron las siguientes variables: edad decimal a través de la fecha de examen y la fecha de nacimiento, sexo, grupo racial, datos socioeconómicos y de morbilidad y un conjunto de dimensiones antropométricas entre las que se encontraban: peso, longitud en decúbito supino (menores de tres años), estatura 
(dos años o más), circunferencia cefálica (hasta los 6 años), circunferencia del brazo izquierdo extendido, circunferencia de la cintura ( 8 años o más), circunferencia de la cadera (8 años o más), pliegue cutáneo tricipital izquierdo, pliegue cutáneo subescapular izquierdo, pliegue cutáneo suprailíaco izquierdo y la menarquía (niñas de 8 o más años). ${ }^{7}$

En este trabajo se presentan los resultados de la comparación del peso y la talla de los niños con las nuevas referencias internacionales de la OMS (WHO/MGRS) a partir del análisis del comportamiento de los indicadores peso para la edad, talla para la edad, peso para la talla e índice de masa corporal (IMC) para la edad. Se estimaron, para cada niño, las puntuaciones $Z$ de cada uno de esos indicadores, usando para ello el software WHO Antro, $2005^{8}$ provisto por la OMS para este fin. Se calcularon las medias y desviaciones estándar (DE) de estas puntuaciones para toda la población y según grupos de edad y sexo y se estimaron las tasas de prevalencia de insuficiencia ponderal, cortedad de talla y emaciación mediante el cálculo de los porcentajes de niños con peso para la edad, talla para la edad, peso para la talla e IMC para la edad por debajo del valor límite de - 2 puntuaciones z del MGRS. También se obtuvo la frecuencia del sobrepeso en esta población mediante la obtención de los porcentajes de preescolares con peso para la talla e I MC para la edad por encima de 2 puntuaciones $z$ de la referencia. Se compararon estos resultados con los que se obtendrían con el uso de los valores del NCHS y con las normas nacionales cubanas.

\section{RESULTADOS Y DISCUSIÓN}

La tabla 1 informa sobre la distribución de los niños estudiados según grupos de edad y sexo.

Tabla 1. Distribución de los niños según edad y sexo

\begin{tabular}{|l|c|c|c|}
\hline $\begin{array}{c}\text { Grupo de } \\
\text { edad } *\end{array}$ & Niños & Niñas & Total \\
\hline $0-5$ & 78 & 71 & 149 \\
\hline $6-11$ & 94 & 81 & 175 \\
\hline $12-23$ & 144 & 160 & 304 \\
\hline $24-35$ & 157 & 145 & 302 \\
\hline $36-47$ & 108 & 100 & 206 \\
\hline $48-60$ & 95 & 91 & 186 \\
\hline Total & 674 & 648 & 1322 \\
\hline
\end{tabular}

*: Edad en meses.

En la tabla 2 se observan los valores de las medias y desviaciones estándar de las puntuaciones $Z$ de los indicadores seleccionados para el conjunto de la población estudiada.

Tabla 2. Medias y desviaciones estándar de las puntuaciones $Z$ de los indicadores antropométricos seleccionados 


\begin{tabular}{|l|c|c|}
\hline \multicolumn{1}{|c|}{$\begin{array}{c}\text { Indicador } \\
\text { antropométrico }\end{array}$} & \multicolumn{2}{c|}{ Puntuación Z } \\
\cline { 2 - 3 } & Media & DE \\
\hline Peso/edad & 0,33 & 1,12 \\
\hline Talla/edad & $-0,07$ & 1,08 \\
\hline Peso/talla & 0,50 & 1,16 \\
\hline I MC/edad & 0,51 & 1,16 \\
\hline
\end{tabular}

Puede observarse que en el caso de los tres indicadores que involucran el peso de los niños los valores medios de las puntuaciones resultaron positivos, oscilando en el rango entre 0,33 y 0,51, sólo la talla para la edad mostró un valor negativo de 0,07 . Nótese que las desviaciones estándar de la distribución de las puntuaciones siempre fueron superiores a 1 pero inferiores a 1,2 .

Los autores del MGRS, al evaluar las diferencias en el crecimiento longitudinal de las poblaciones que integraron la muestra de ese estudio, aceptaron el criterio de Cohen acerca de que diferencias de 0,2 DE pueden ser consideradas como pequeñas, 0,5 como medianas y 0,8 como grandes ${ }^{9}$ y decidieron en base a ello que los datos de los diferentes grupos poblacionales podían ser mezclados si las diferencias eran menores que medianas, o sea, inferiores a $0,5 .{ }^{10}$

De acuerdo con esto, las diferencias entre los niños habaneros estudiados y la población de referencia pueden catalogarse como medianas en el caso de los indicadores peso para talla e IMC para la edad, mientras que en cuanto al crecimiento longitudinal serían pequeñas. El peso para la edad tendría un comportamiento intermedio, aunque sus diferencias clasificarían como medianas. En resumen, estos resultados hablarían de una población infantil con una relación peso/talla superior a la de la referencia pero con un menor crecimiento en talla, comportamiento similar al encontrado en poblaciones opulentas de Argentina e Italia. ${ }^{11}$

Es clásico el trabajo de Habicht en el que señala que no existen diferencias relevantes en las medias de talla entre niños preescolares de diferentes grupos étnicos si estos se desarrollan en condiciones ambientales favorables. ${ }^{12}$ Los autores del MGRS arribaron también a una conclusión similar. ${ }^{10}$ Si se asume esta afirmación, los resultados obtenidos en la talla de los niños estudiados podrían considerarse como una expresión del bienestar y la calidad de vida de esta población infantil. En relación con las diferencias en el peso para la edad y los dos indicadores que evalúan la relación peso/talla pueden existir diversos factores pero, evidentemente, uno de ellos está relacionado con las diferencias en la alimentación de los niños estudiados respecto a la referencia ya que mientras que los estándares de la OMS se basan únicamente en niños "amamantados", en Cuba sólo el $26 \%$ de los lactantes menores de seis meses utilizan lactancia materna exclusiva según los resultados de la Encuesta MICS, realizada por el Ministerio de Salud Pública (MI NSAP) en colaboración con el Fondo de Naciones Unidas para la Infancia (UNICEF) durante el año 2006. ${ }^{13}$

La figura 1 muestra el comportamiento de las puntuaciones Z medias de los cuatro indicadores analizados en comparación con los datos de referencia de WHO/MGRS según sexo y edad. Obsérvese que la forma de las curvas es similar para ambos sexos en los cuatro indicadores analizados y que, en el caso del peso para la edad, 
los valores más altos se alcanzaron en los grupos de 6 a 11 y 12 a 23 meses en que llegaron a cifras muy cercanas a 0,5 ; presentando las niñas valores superiores a los del sexo masculino excepto en los grupos extremos. Este último comportamiento también se observó en la talla para la edad pero, a diferencia del peso, esta dimensión muestra valores negativos de las medias de las puntuaciones $Z$ hasta el grupo de 12 a 23 meses en las niñas y en todos los grupos de edad del sexo masculino, excepto en el de 48 a 60 meses; en ambos casos, el indicador mostró una tendencia ascendente a medida que avanzó la edad de los niños.

El peso para la talla y el I MC para la edad presentaron, como era de esperar, un patrón similar, con una curva que asciende bruscamente entre el grupo de 0 a 5 meses y el de 6 a 11 en el caso de los niños y hasta los 12 a 23 meses en el caso de las niñas, por lo que llegaron a ser elevadas las diferencias entre esta población y la referencia de la OMS pues alcanzaron valores de 0,88 y 0,74 y de 0,75 y 0,80 para niños y niñas en el caso del peso para la talla y de 0,86 y 0,81 en el sexo masculino y 0,71 y 0,86 en el femenino para el IMC para la edad durante la segunda mitad del primer año y el segundo año de vida; esto supera el criterio de Cohen de considerar como diferencias elevadas aquellas con magnitudes superiores a 0,8 . Ello puede ser una expresión de las diferencias ya mencionadas en la alimentación de estos niños y que, de ser así, se constituiría en un reto a resolver por el sistema de salud para evitar efectos nocivos para su salud desde etapas tempranas de la vida. Los niños siempre mostraron cifras de $Z$ superiores en estos dos últimos indicadores a las del sexo femenino, excepto en el grupo de 12 a 23 meses.

Sólo con la excepción del grupo de 12 a 23 meses, los niños siempre mostraron cifras de $Z$ superiores en estos dos últimos indicadores a las del sexo femenino.

Hay un hecho llamativo en la observación de estos gráficos: las tendencias de las curvas cambian su comportamiento, de manera abrupta, a partir de los dos años. En los indicadores relacionados con el peso, las tendencias son ascendentes hasta esa edad $y$, bruscamente adquieren una tendencia descendente, mientras que en la talla ocurre la situación inversa, cifras iniciales negativas y, por ende, bajas de las puntuaciones $Z$, son seguidas después de los dos años por valores cercanos a cero o francamente positivos.

¿Será expresión este fenómeno de cambios en la situación nutricional y de salud de estos niños a partir de esa edad o estará relacionado con las diferencias metodológicas entre los dos componentes de la referencia de la OMS? No hay ningún elemento plausible que indique que en la población estudiada pueda haber ocurrido ninguna situación relevante que haya modificado de manera tan notoria el comportamiento de los patrones de crecimiento de los niños de mayor edad respecto a los más pequeños. Por otra parte, los valores del MGRS proceden de un estudio longitudinal en los primeros 24 meses mientras que, posteriormente, fue continuado por un estudio transversal de niños entre 18 y 71 meses. ${ }^{6}$ Aunque esto permitió solapar ambas investigaciones en el segundo semestre del segundo año de vida, parecería verosímil inferir que los niños del componente longitudinal mostraron diferencias en su crecimiento respecto a los del componente transversal que pueden haber influido en estos resultados.

La figura 2 presenta los porcentajes de preescolares con peso para la edad, talla para la edad, peso para la talla e IMC para la edad por debajo del valor límite de - 2 puntuaciones $z$ en comparación con los datos de referencia de WHO/MGRS. Serían estos los niños que clasificarían, de acuerdo con el indicador, con afectaciones nutricionales moderadas a severas. Las prevalencias globales de insuficiencia ponderal, cortedad de talla y emaciación -ya fuera con el uso del peso para la talla 
o el I MC para la edad- fueron de 1,3; 3,7; 1,0 y 1,0 \%, respectivamente. Los niños siempre se mostraron mas afectados que las niñas. Sólo en el caso de la talla para la edad se produjo un porcentaje superior al 2,3\% esperado (ya que se está utilizando el valor de $-2 \mathrm{DE})$.

Cuando se analizó, en la figura 3 , el comportamiento de la situación nutricional de los niños considerando su edad, se pudo observar que la menor prevalencia de insuficiencia ponderal se presentó en el grupo de 12 a 23 meses y que la edad de 36 a 47 meses fue la mas afectada, con una prevalencia de 2,9\%; las niñas mostraron porcentajes superiores de bajo peso en el grupo de edad inicial y, también, aunque muy discretamente, en los dos grupos finales, pero, sin embargo, no se encontraron casos en esta situación en las edades de 6 a 11 y 12 a 23 meses en este sexo.

La afectación de la talla para la edad fue la situación deficitaria más común, con valores porcentuales que se mantuvieron en cifras que oscilaron entre 3,3 y 4,9\%, con la única excepción del grupo de 48 a 60 meses en el que la prevalencia de baja talla fue solo de $1,0 \%$. En cuanto al peso para la talla y el IMC para la edad, los porcentajes de niños con emaciación moderada a severa mostraron cifras muy bajas, con excepción del grupo de 36 a 47 meses que alcanzó una cifra de 3,4 \%, tanto en el caso del peso para la talla como del IMC para la edad. Estos últimos resultados indicaron que la prevalencia de bajo peso para la edad de las primeras edades responde fundamentalmente a la baja talla y no a la emaciación.

Es de resaltar que aunque las desviaciones negativas del crecimiento de lactantes amamantados que se desarrollaban en condiciones favorables respecto a los datos del NCHS/OMS fue uno de los elementos más importantes que argumentaron la necesidad de una nueva referencia, ${ }^{14}$ ahora aparece, como un hallazgo importante del MGRS, que los niños que conformaron su muestra y que se caracterizaron por este tipo de lactancia fueron, como promedio, más altos que los que conformaron la del NCHS y, también, que los valores publicados por el estudio CDC 2000 de Estados Unidos. Con esto, señalaban, se disiparon las preocupaciones relacionadas con que los niños amamantados podrían fallar en alcanzar su potencial para el crecimiento del tejido magro debido a ingestas marginales de energía, proteína y otros nutrientes. ${ }^{15}$

No obstante, ahora parecen surgir otras inquietudes, vinculadas esta vez con el incremento que este comportamiento de los nuevos patrones producirá en las tasas de prevalencia del retardo del crecimiento de los niños en el mundo y con el hecho de si lograr estos valores será una meta realista, ya que deberían alcanzar un crecimiento longitudinal que ni poblaciones como la de Estados Unidos ha logrado obtener. Adicionalmente, la homogeneidad de la muestra del MGRS induce a que la distribución de los valores sea más estrecha de lo que sería en una población más heterogénea, lo que da como resultado valores límites más cercanos a la mediana, hecho que también puede influir en los resultados de la evaluación nutricional.

Las figuras 4 y $\underline{5}$ presentan los porcentajes de niños que mostraron cifras superiores a 2 DE de los indicadores peso para talla e IMC para edad, es decir, aquellos que clasificaron con sobrepeso moderado a severo. EI IMC mostró valores porcentuales de niños con cifras por encima del valor límite de 2 puntuaciones $z$ ligeramente superiores que el peso para la talla ( $11,2 \%$ vs $10,7 \%)$. Los niños presentaron sobrepeso con más frecuencia que las niñas y los grupos de edad mas afectados fueron los de 6 a 11 y 12 a 23 meses.

Es una realidad que los resultados de la evaluación del crecimiento y estado de nutrición de los niños depende en gran medida, de la referencia utilizada. En las 
figuras 6 y $\underline{7}$ se observan las variaciones en la prevalencia de las diferentes formas de malnutrición en dependencia del uso de los valores de WHO/MGRS, WHO/NCHS o de las normas nacionales cubanas. La prevalencia de bajo peso para la talla se incrementó con el uso de las normas nacionales mientras que la de retardo del crecimiento disminuyó considerablemente, el sobrepeso aumentó con el uso de WHO/MGRS. Las diferencias encontradas son importantes $y$, por ende, también lo son las implicaciones que ello tiene sobre las políticas y estrategias a desarrollar.

Los autores del MGRS ya habían alertado acerca de los cambios que ocurrirían en las tasas de prevalencia de la malnutrición como consecuencia del uso internacional de la nueva referencia. Ellos señalaron que:

Los estimados cambiarán debido a diferencias en el patrón de crecimiento entre los nuevos estándares y la antigua referencia, especialmente en los lactantes. La magnitud del cambio variará por edad, sexo, indicador de crecimiento y el estado nutricional subyacente en la población a evaluar, y precisaban que el retardo será mayor a través de la infancia, se producirá un incremento sustancial en la tasa de bajo peso durante los primeros seis meses con una disminución posterior, disminuirá la emaciación hasta alrededor de los $70 \mathrm{~cm}$ cuando las tasas serán sustancialmente mayores con los nuevos estándares y habrá una mayor prevalencia de sobrepeso que variará con la edad, sexo y estado nutricional de la población estudiada. ${ }^{16}$

La magnitud de estas discordancias exige ser precavidos al interpretar el crecimiento de esta población. Los niños cubanos son de menor talla y mas pesados que los de los nuevos patrones de la OMS y, también, que los de la antigua referencia del NCHS, lo que se ha demostrado en estudios previos a este. ${ }^{17}$ Por otra parte, aunque el patrón diferente de alimentación puede inducir diferencias en el nivel de adiposidad no siempre los valores excesivos de peso responden a valores elevados de esta, pueden estar relacionados también con otras diferencias en la composición corporal y en las proporciones del cuerpo. ${ }^{18}$

El problema estaría en qué medida las diferentes tasas de prevalencia de malnutrición, obtenidas con el uso de una u otra referencia, realmente expresan, o no, desviaciones patológicas del crecimiento de los niños. Esta es una pregunta crucial ya que, en dependencia de la respuesta, se deberá decidir si se ejercen acciones para modificar el desarrollo físico de aquellos que no encontrándose, por una u otra razón, en las condiciones "ideales" en que ha ocurrido el crecimiento de la población de referencia, alcanzan un fenotipo adaptado a sus condiciones de vida. Sería interesante conocer, y queda como una interrogante para próximos estudios, si las diferencias encontradas se comportan de igual forma en los grupos de niños cubanos que cumplen las orientaciones de lactancia y alimentación complementaria establecidos por la OMS.

\section{REFERENCI AS BI BLI OGRÁFICAS}

1. Organización Panamericana de la Salud. Promoción del crecimiento y desarrollo integral de niños y adolescentes. Serie HCT/AOIEPI-25.E.1.Washington, D.C.: OPA; 1999.

2. de Onis M, Gaza C. Foreword. Acta Paediatrica. 2006; 450:5-6. 
3. de Onis M, Wijnhoven TM, Onyango AW. Worldwide practices in child growth monitoring. J Pediatr. 2004; 144:461-5.

4. WHO Multicentre Growth Reference Study Group. WHO Child Growth Standards based on length/height, weight and age. Acta Paediatr Suppl. 2006;450:76-85.

5. WHO Multicentre Growth Reference Study Group. WHO Child Growth Standards: length/height-for-age, weight-for-age, weight-for-length, weight-for-height and body mass index-for-age: methods and development. Geneva: World Health Organization; 2006.

6. de Onis M, Garza C, Victora CG, Onyango AW, Frongillo EA, Martines J. The WHO Multicentre Growth Reference Study: Planning, study design and methodology.

Food Nutr Bull. 2004;25(Suppl 1):S15-26.

7. Grupo de Crecimiento y Desarrollo. Estudio sobre Crecimiento y Desarrollo de Ciudad Habana, 2005. Informe Final de Investigación. La Habana: Instituto Superior de Ciencias Médicas de La Habana; 2006.

8. WHO Anthro 2005, Beta version Feb 17th, 2006: Software for assessing growth and development of the world's children. Geneva: WHO; 2006. [Disponible en: http://www. who.int/childgrowth/software/en/]

9. Cohen J. Statistical power analysis for the behavioral sciences. 2nd ed. New Jersey: Lawrence Erlbaum Associates; 1988.

10. WHO Multicentre Growth Reference Study. Assessment of differences in linear growth among populations. Acta Pædiatri. 2006; (Suppl 450):56-65.

11. Oyango AW, de Onís M, Caroli M, Shah U, Sguassero Y, Redondo N, et al. FiedTesting the WHO child growth standards in four countries. J Nutr.

2007; 137: 149152.

12. Habicht JP, Martorell R, Yarbrough C, Malina RM, Klein RE. Height and weight standards for preschool children: How relevant are ethnic differences in growth potential? Lancet. 1974; 1:611-4.

13. Dirección Nacional de Estadísticas MINSAP. Cuba. Encuesta de Indicadores Múltiples por Conglomerados. La Habana: DNE, MINSAP;2006.

14. World Health Organization. Physical status: the use and interpretation of anthropometry. Report of a WHO Expert Committee. Technical Report Series No. 854 .Geneva: World health Organization; 1995.

15. de Onis M, Garza C, Onyango AW, Borghi E. Comparison of the WHO Child Growth Standards and the CDC 2000 Growth Charts. J Nutr. 2007; 137: 144-8.

16. World Health Organization. Frequently asked questions. Do these new standards change current estimates of overweight and under-nutrition in children? [citado 22 Ago de 2008]. Disponible en: http://www.who.int/childgrowth/faqs/en/

17. Esquivel M, Romero J M, Berdasco A, Gutiérrez J, Jiménez J M, Posada E, et al. Estado nutricional de preescolares de Ciudad de La habana entre 1972 y 2003 . Rev Panam Salud Pública. 1997; 1(5):349-54. 
18. Trowbridge FL, Marks JS, Lopez de Romana L. Body composition of Peruvian children with short stature and height weight-height. II: Implications for the interpretation for weight for height as an indicator of nutritional status. Am J Clin Nutr. 1987;46:411-8.

Recibido: 2 de septiembre de 2008.

Aprobado: 21 de octubre de 2008.

Mercedes Esquivel Lauzurique. Departamento de Crecimiento y Desarrollo Humano. Facultad de Ciencias Médicas "Julio Trigo López". Instituto Superior de Ciencias Médicas de La Habana. La Habana, Cuba.

E-mail: mesqui@infomed.sld.cu

Peso para la edad

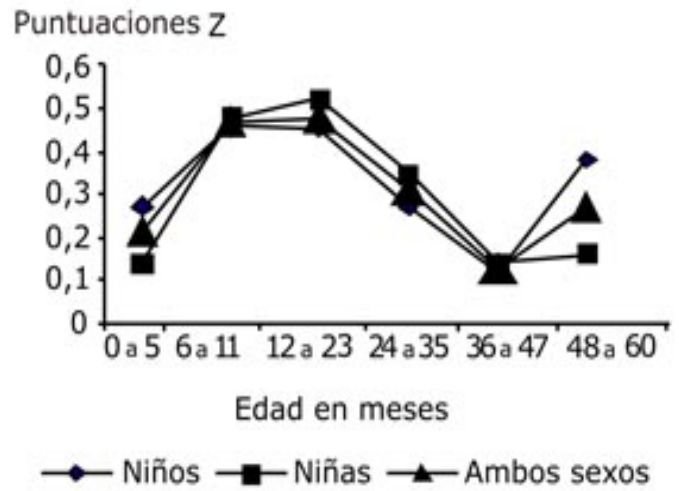

Peso para la talla

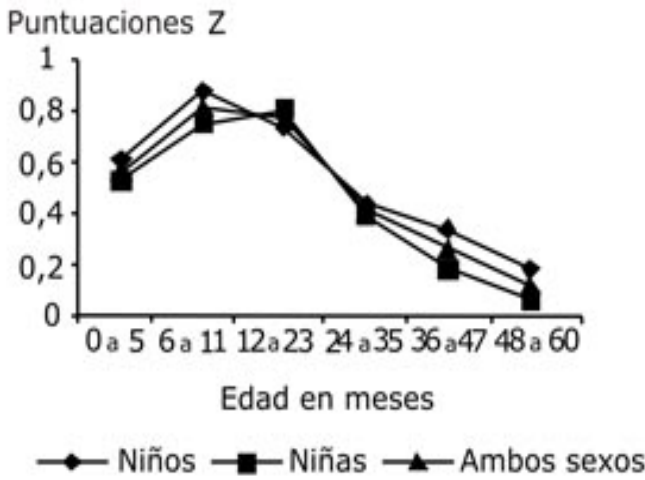

Talla para la edad

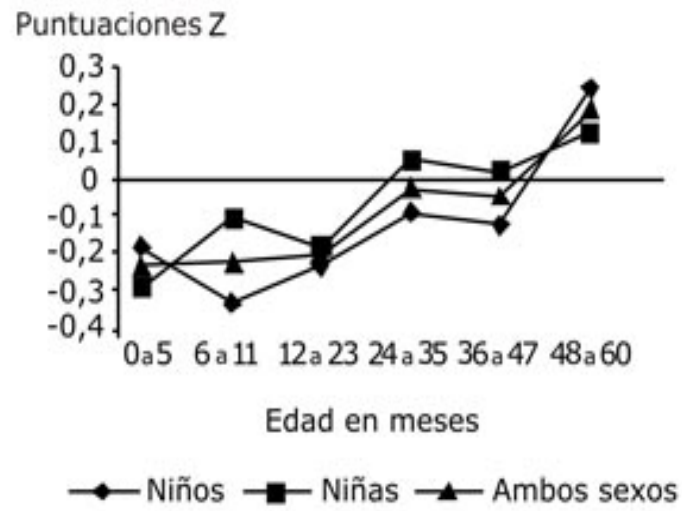

IMC para la edad

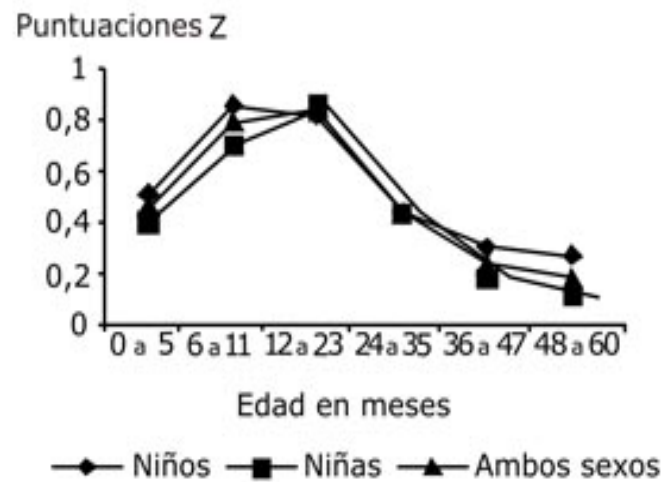

Fig.1.Puntuaciones $Z$ medias de los indicadores peso para la edad, talla para la edad, peso para la talla e IMC para la edad en comparación con los datos de referencia de WHO/MGRS según sexo y grupo de edad. 


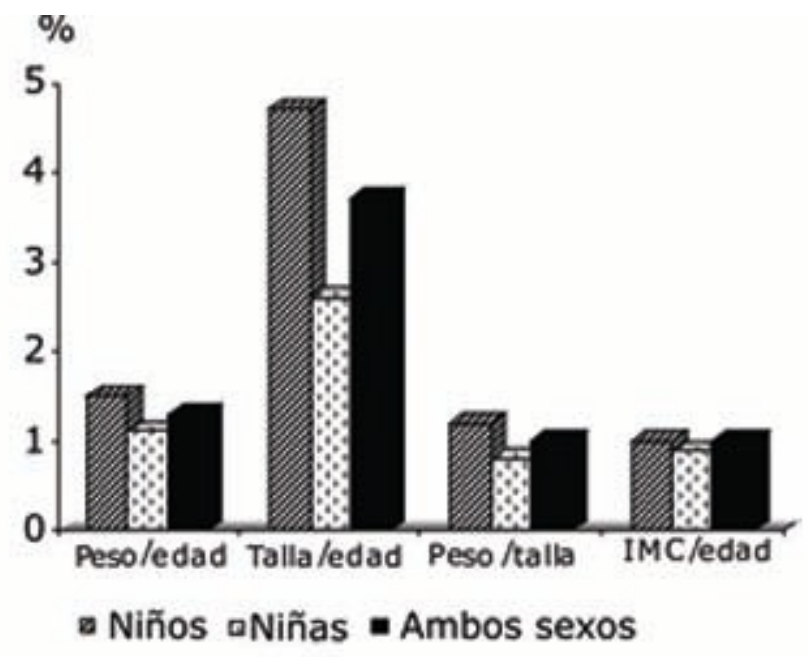

Fig.2. Porcentaje de preescolares con peso para la edad, talla para la edad, peso para la talla e IMC para la edad por debajo del valor límite de -2 puntuaciones $z$ en comparación con los datos de referencia de WHO/MGRS.

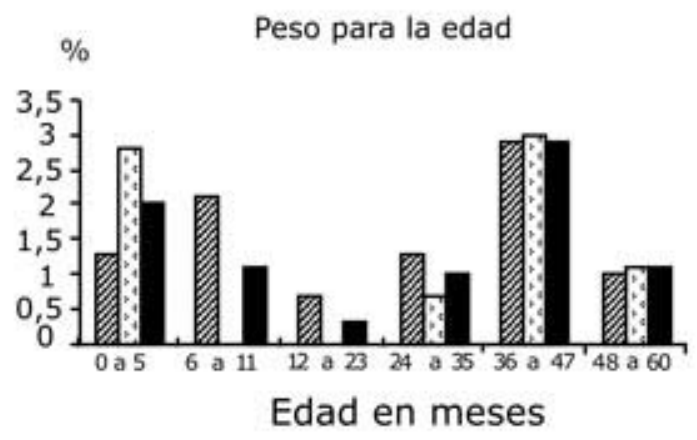

๒Niños घNiñas —Ambos sexos

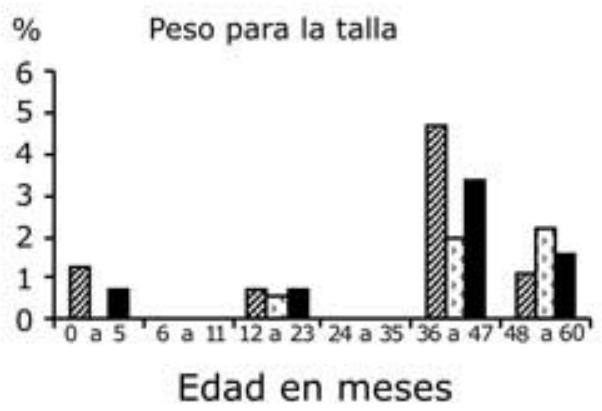

๒Niños ๒Niñas

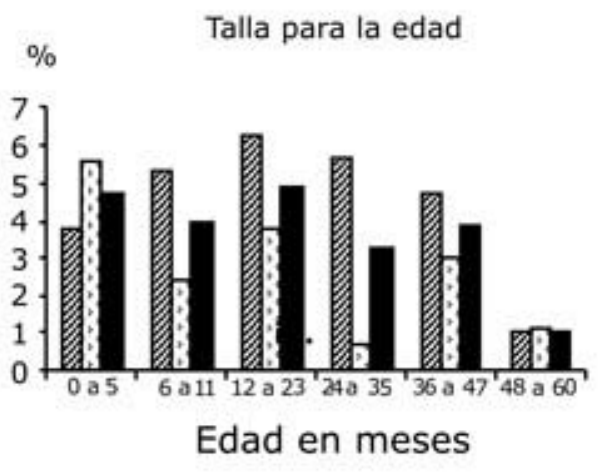

खNiños घ Niñas Ambos sexos

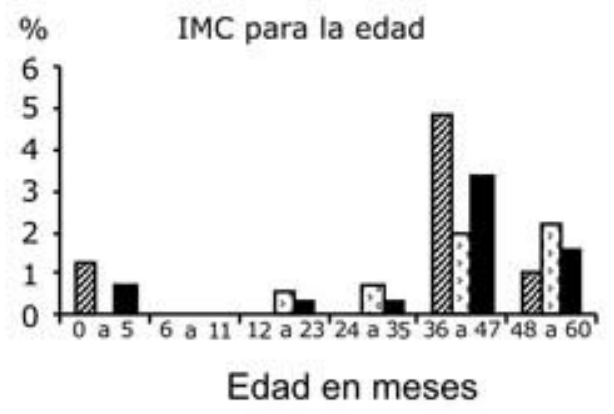

๒ Niños Niñas Ambos sexos

Fig. 3. Porcentaje de niños con peso para la edad, talla para la edad, peso para la talla e IMC para la edad por debajo del valor limite de -2 puntuaciones z en comparación con los datos de referencia de WHO/MGRS según sexo y edad. 


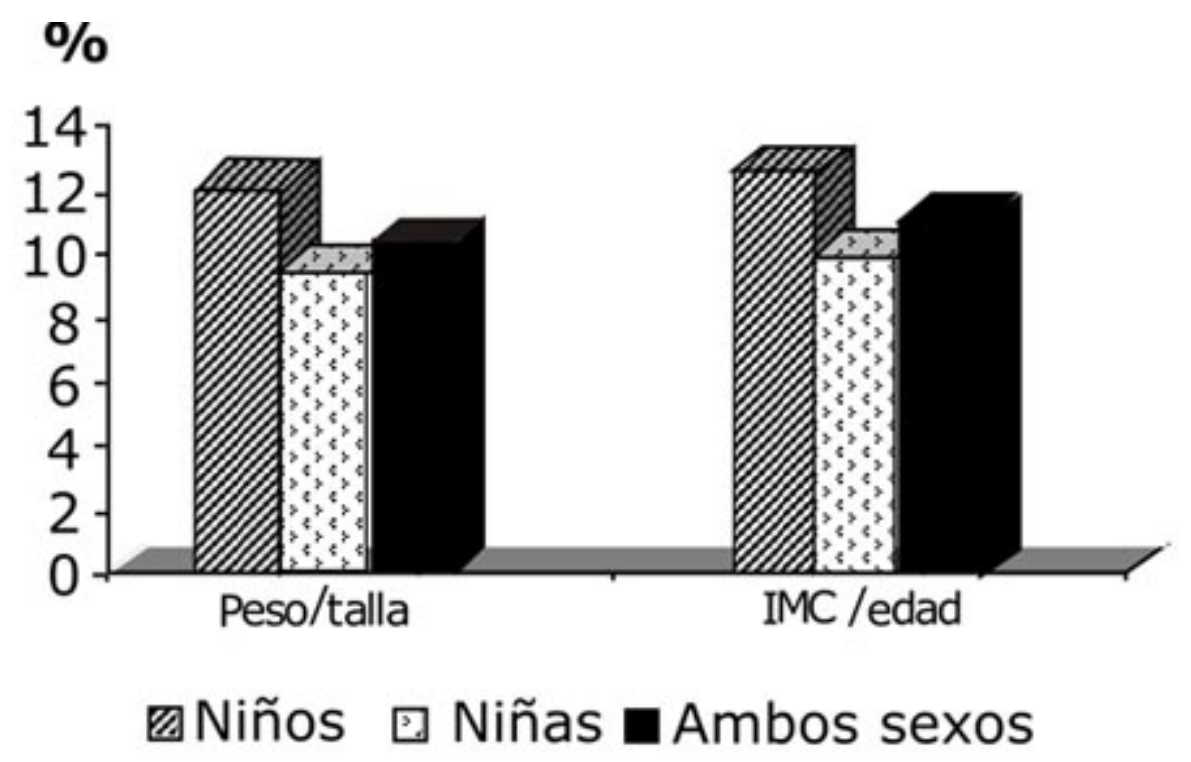

Fig. 4. Porcentaje de preescolares con peso para la talla e IMC para la edad por encima de 2 puntuaciones $z$ en comparación con los datos de referencia de WGRS.

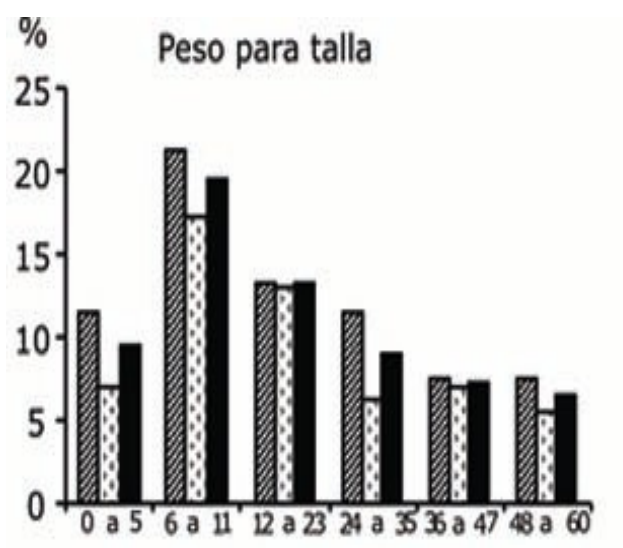

Edad en meses

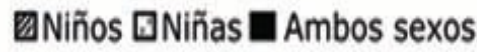

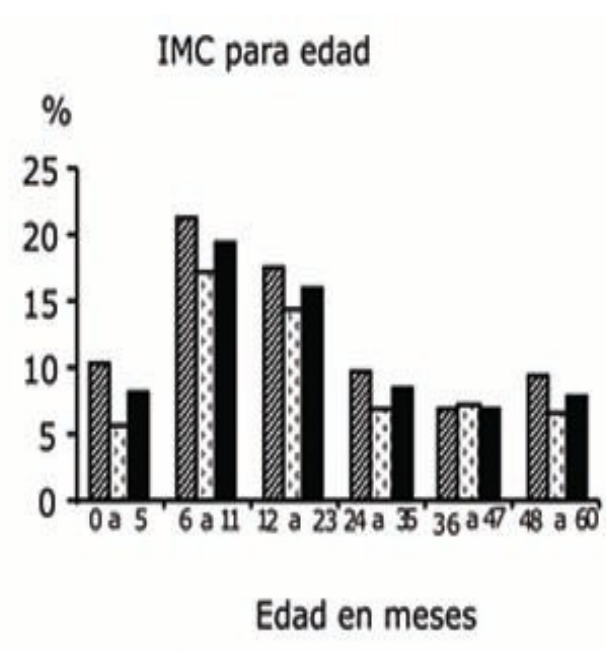

圆 Niños 曰Niñas —Ambos sexos

Fig. 5. Porcentaje de preescolares con peso para la talla e IMC para la edad por encima de 2 puntuaciones z en comparación con los datos de referencia de WHO/MGRS según edad. 


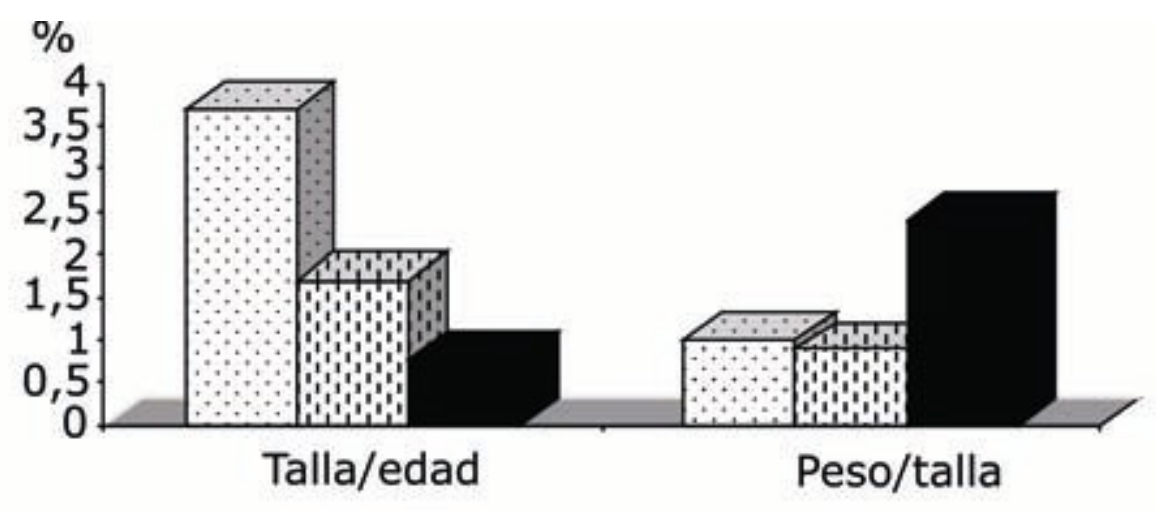

$\square$ WHO/MGRS * $\square$ WHO/NCHS * घCUBA **

* Niños por debajo de -2 DE en el caso de WHO/MGRS y WHO/NCHS, ** Niños por debajo del tercer percentil en el caso de las normas cubanas.

Fig. 6. Porcentaje de niños con afectación nutricional moderada a severo según indicadores y patrones de referencia.

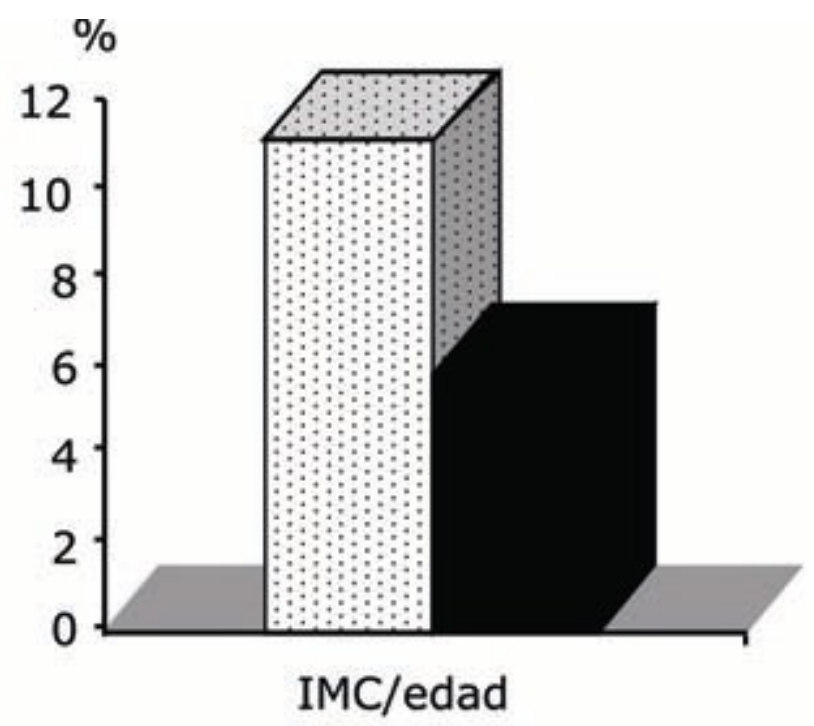

圆WHO/MGRS $*$ CUBA $* *$

* Niños por encima de 2DE en el caso de MGRS,

**Niños por encima del percentil 97 en el caso de las normas cubanas.

Fig. 7. Porcentaje de niños con sobrepeso moderado a severo según indicadores y patrones de referencia. 